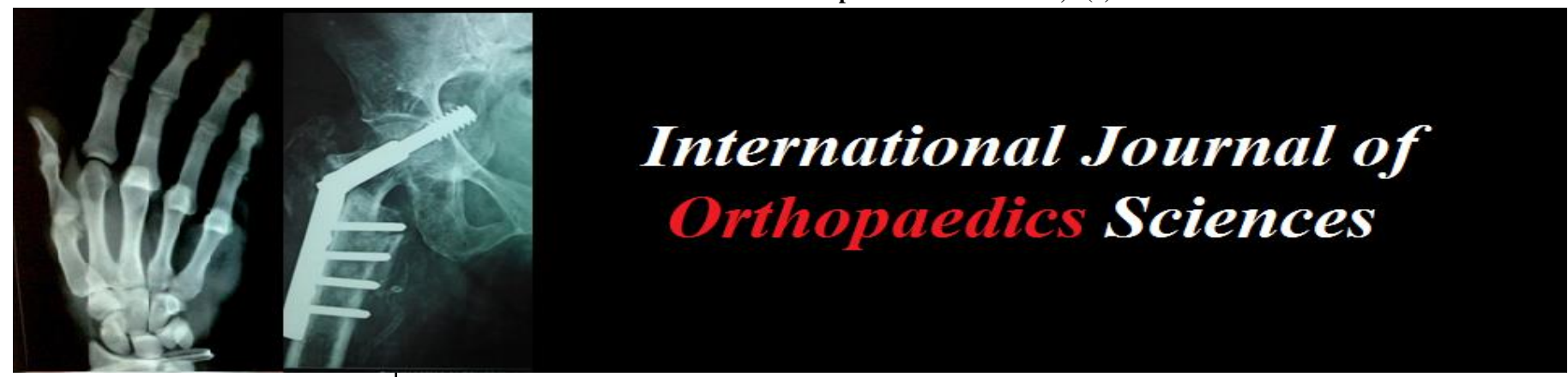

ISSN: $2395-1958$

IJOS 2018; 4(2): 742-744

(C) 2018 IJOS

www.orthopaper.com

Received: 17-02-2018

Accepted: 18-03-2018

\section{A Bhandari}

Department of Orthopaedics, HIMS, Swami Rama Himalayan University, Dehradun

Uttarakhand, India

\section{AKS Deane}

Department of Orthopaedics, HIMS, Swami Rama Himalayan University, Dehradun,

Uttarakhand, India

\section{Correspondence}

A Bhandari

Department of Orthopaedics,

HIMS, Swami Rama Himalayan

University, Dehradun,

Uttarakhand, India

\section{Evaluation of the neck shaft angle achieved after surgical fixation of intertrochanteric fracture of femur}

\section{A Bhandari and AKS Deane}

DOI: https://doi.org/10.22271/ortho.2018.v4.i2k.107

\section{Abstract}

Introduction: Femoral trochanteric fractures are the most common fractures of old age. Due to the fracture there is a change in the neck shaft angle which if not restored can lead to further disability. In this study we evaluated the immediate post-operative neck shaft angle achieved in surgically fixed intertrochantric fracture of femur using DHS and PFN and compared it with the normal side.

Material \& Method: This observational study was performed on 30 patients, with a mature skeleton, with an intertrochantric fracture of femur who got operated within a period of one year. AP view of Xray pelvis with bilateral hip in 15 degree internal rotation was done in the supine position after surgery. Morphometric measurements bilaterally on digital X-ray machine (CR system) using DIACOM software were done. All the data was analyzed for evaluation of neck shaft angle in non-fractured side and the restoration/change in neck shaft angle on the operated side after surgical fixation.

Result: In our study the average neck shaft angle of normal side of femur was found to be $121.17 \pm 6.88$ degrees and that of the operated side was found to be $125.32 \pm 10.12$ degrees.

Discussion: With DHS we were not able to restore the anatomical NSA due to the valgus reduction but with PFN we were able to restore.

Keywords: Neck shaft angle achieved, surgical fixation, intertrochanteric fracture, femur

\section{Introduction}

The Neck-shaft angle (NSA) of femur is the angle between the axis of the femoral shaft and femoral neck ${ }^{[1]}$.

Hip surgery is the commonest surgical procedure, done to restore the NSA. Evaluation of the NSA of femur helps in understanding biomechanics of the hip joint and its clinical relevance and it also helps in planning of treatment of various affections of hip region and in designing implants and prosthesis for this region.

NSA has been shown to vary between different races. The anthropometric dimensions described as normal for proximal end of femur for western region may be quite different from these encountered amongst Indian population ${ }^{[2]}$.

\section{Materials and Methods}

The study was carried out in the Department of Orthopedics at Himalayan Institute of Medical Sciences, Swami Ram Nagar, Dehradun over a period of 12 months. 30 Patients with Intertrochanteric fracture which satisfied the inclusion criteria were taken for our study.

Exclusion criteria:

1. Patients with bilateral intertrochanteric fracture.

2. Previously operated cases of fracture neck of femur or intertrochanteric fracture.

3. Patients who had any condition affecting NSA in non-fractured limb e.g. congenital deformity (DDH), Perthe's disease and AVN of head of femur.

Evaluation of the functional outcome of the surgery was not the part of this study.

\section{Study Protocol}

1. X-ray of pelvis with both hips Antero-posterior view was taken after fixation of fracture using the standardized protocol in 15 degrees of internal rotation of the hips in the supine 
position with a film-focus distance of $100 \mathrm{~cm}$, and the beam centered on the symphysis pubis under our supervision.

2. Measurement of NSA using DIACOM software was done on digital X-ray on postoperative films.

3. Data was then analyzed for NSA in non-fractured limb and the restoration/change in NSA in operated hip after surgical fixation.

4. Use of implant was at the discretion of the surgeon and no randomization or criteria for implant use were assigned beforehand.

\section{Methods}

The data was analyzed with SPSS software version 20.0. Interpretation and analysis of obtained results was carried out using descriptive statistics.

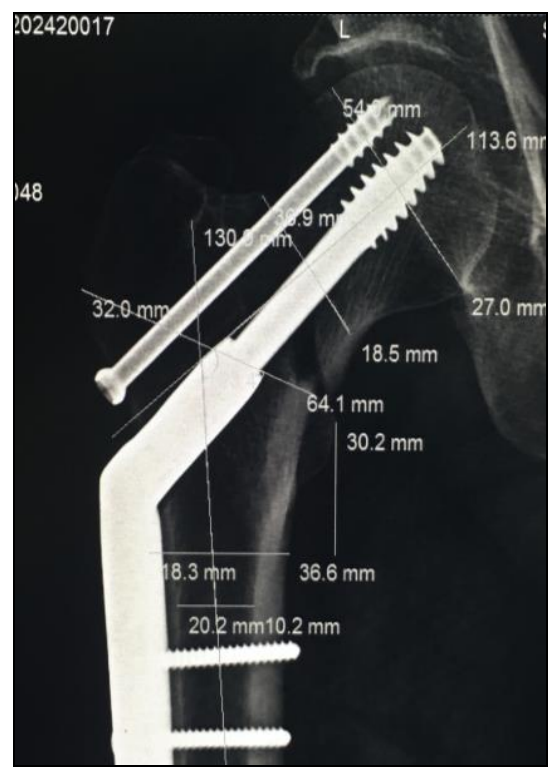

\section{Discussion}

In our study, the statistical analysis showed the mean neck shaft angle of normal side 122.39 degrees and angle restored after Dynamic Hip Screw fixation (DHS) was 129.81 degree. The mean neck shaft angle of normal side was 119.45 degrees and angle restored after Proximal Femoral Nail (PFN) was 119.42 degrees, which is in co-relation with the result of the study conducted by Pajarien $\mathrm{J}$ et al.

They had similar result where they measured from the uninjured and injured hip in 48 patients with a pertrochanteric femoral fracture treated with the Dynamic Hip Screw (DHS) or the Proximal Femoral Nail (PFN). In DHS group the mean NSA of normal side was 128.0 degrees and angle restored after surgery was 134.5 degrees. In PFN group the mean neck shaft angle of normal side was 125.5 degrees and angle restored after surgery was 125.3 degrees. The most important finding of their series was that the mean NSA angle decreased in both treatment groups. This decrease was more distinct in the DHS group, but the difference in patients treated with the PFN was not significant ${ }^{[3]}$.

In another study conducted by Barwar $\mathrm{N}$ et al. showed that there is a high incidence of coxa vara or coxa valga in fixation with standard dynamic hip screw and restoration of NSA could often not be achieved. Our results are comparable with their study ${ }^{[4]}$.

The information on proximal femoral morphology presented in this study could be used as a guideline to design a more suitable implant for the Indian population, which covers most
Student t- test was used for comparison of various parameters pertaining to continuous data.

\section{Results}

A total of 30 patients with mature skeleton were selected with the youngest being 35 years of age and the oldest 90 years of age. 19 of the patients were males and 11 were females. All of the patients were operated and DHS was applied as an implant in 18 of the cases and PFN in 12 of them.

The mean NSA of the normal side was 122.39 \pm 7.99 degrees and on the operated side was $129.81 \pm 6.88$ degrees after DHS and the $\mathrm{p}$ value was found to be 0.006 , which is significant. While the mean NSA of normal side was 119.45 \pm 5.13 degrees and on the operated side after PFN fixation was $119.42 \pm 11.37$ degrees and the $p$ value here was found to be 0.99 , which is not significant.

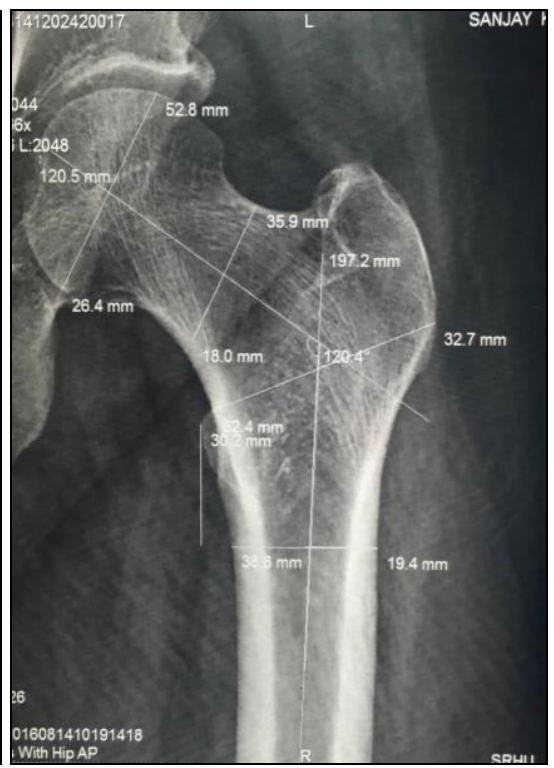

South East Asian countries. The design of hip implant has evolved drastically as lessons were learnt from the many and various post-surgical complications.

With this study we came to the conclusion that by using the DHS as a fixation implant for Intertrochanteric fractures we were not able to restore the anatomical NSA possibly due to the valgus reduction required when using DHS with 135 degrees angled barrel plate but on the other hand with PFN we were able to restore the anatomical NSA possibly because the positioning of the nail and consequently the proximal screws positioning can be varied.

Though outside the domain of this study we strongly recommend the use of a cephalomedullary implant for Intertrochanteric fracture fixation to restore the normal anatomical NSA which may result in better functional result.

\section{Conclusion}

In the present study titled "Evaluation of the neck shaft angle achieved after surgical fixation of intertrochanteric fracture of femur" we have concluded the following:

- With DHS we were not able to restore the anatomical NSA possibly due to the valgus reduction.

- With PFN we were able to restore the anatomical NSA.

\section{References}

1. Pujari R, Shankar RG, Naveen NS, Roopa CR. Evaluation of Neck Shaft Angle of Femur on Dry Bones. Journal of Evolution of Medical and Dental Sciences. 
2015; 4(32):5518-22.

2. Gujar S, Vikani S, Parmar J, Bondre KV. A correlation between femoral neck shaft angle to femoral neck length. International journal of Biomedical and Advanced Research. 2013; 04:5-11.

3. Pajarinen J, Lindahl J, Savolainen V, Michelsson O, Hirvensalo E. Femoral shaft medialisation and neck-shaft angle in unstable pertrochanteric femoral fractures. International Orthopedics. 2004; 28:347-53.

4. Barwar N, Meena S, Aggarwal SK, Garhwal P. Dynamic hip screw with locking side plate: a viable treatment option for intertrochanteric fracture. Chinese Journal of Traumatology. 2014; 17(2):88-92. 